\title{
Intérêt comparé du traitement à l'ammoniac et d'une complémentation appropriée de pailles de blé (niveau et nature des compléments énergétiques et azotés) pour l'alimentation de génisses de race laitière de deux ans en croissance hivernale modérée
}

\author{
M. Chenost \\ avec la collaboration technique de J.M. Boissau, L. L'Hôtelier et J.P. Chaise
}

INRA, unité de valeur alimentaire, Theix, 63122 Ceyrat, France

(reçu le 22 juin 1988, accepté le 3 mars 1989)

Résumé - Des pailles de blé en l'état, P.NT, ou traitées à l'ammoniac, P.NH $\mathrm{N}_{3}$ (au four FMA ou en meule à raison, respectivement, de 30 et $50 \mathrm{~g} \mathrm{~d} \mathrm{NH}_{3}$ par $\mathrm{kg}$ de paille) ont été distribuées à des génisses laitières de race Pie Noire, âgées de 2 ans, pendant 3 hivers successifs (essais I, II et III). Les P.NT et P.NH $\mathrm{H}_{3}$ étaient toutes complémentées par $1,5 \mathrm{~kg}$ de matière sèche d'ensilage d'herbe et par 2,6 (P.NT) et 2,6 - 2,0 - 0,9 - ou $0 \mathrm{~kg}$ de MS/j (P.NH $\mathrm{N}_{3}$ ) de pulpes de betteraves déshydratées. 90 $\mathrm{g} / \mathrm{j}$ d'urée étaient ajoutés aux P.NT pour amener leur teneur en équivalent MAT à une valeur voisine de celle $(9,5$ à $11 \%)$ des P.NH ${ }_{3}$. Du tourteau de soja tanné a été apporté à certains lots des essais II et III en quantité excédentaire par rapport aux besoins théoriques en PDI, couverts pour les croissances visées $(350 \mathrm{~g} / \mathrm{j})$.

L'augmentation intéressante de la teneur en équivalent MAT, de la digestibilité et de l'indigestibilité (sur moutons) des pailles, montre que les traitements en four et en meule ont été aussi efficaces les uns que les autres.

Les quantités de P.NT ingérées par les génisses, variant de $2,81 \mathrm{~kg}$ (valeur très faible) à $4,36 \mathrm{~kg}$ de $M S / j$, ont augmenté, après traitement, d'une quantité d'autant plus importante que les rations contenaient moins de pulpes (augmentation allant de 30 à $85 \%$ ). Les croissances des génisses ont toutes été au moins égales (à une exception près) à celles visées. A complémentation en pulpes égale ces croîts sont passés de 218 à 540 (meule) et à $441 \mathrm{~g} / \mathrm{j}$ (four), l'augmentation de la valeur énergétique nette calculée des pailles traitées n'ayant toutefois été que nulle ou de $0,17 \mathrm{UFL} / \mathrm{kg}$ (essai 1). Le traitement a permis de réduire la complémentation énergétique de 1,8 - 1,9 UFL/j tout en maintenant les croissances des génisses. L'augmentation de la valeur énergétique calculée des pailles (qui représentent alors en moyenne $65 \%$ de la ration) est de l'ordre de 0,20 UFL $/ \mathrm{kg}$, la même que celle estimée à partir de la digestibilité mesurée sur moutons.

L'apport d'une source de P.DIA (tourteau tanné), en excès par rapport aux besoins théoriques, n'a pas eu d'effet marqué sur les quantités de pailles ingérées, mais a permis d'augmenter les croîts réalisés avec les P.NT à une valeur voisine de ceux réalisés avec les $\mathrm{P} . \mathrm{NH}_{3}$ (essais II et III) avec moins de complément. II a permis d'augmenter non significativement mais de façon intéressante les croîts réalisés avec les P.NH $\mathrm{N}_{3}$ dans 1 cas sur 2 (essai II).

Ces essais montrent ainsi limportance d'une source d'azote peu et lentement dégradable en complément de P.NT ou de $\mathrm{P.NH}_{3}$, vérifiée jusqu'ici seulement à l'échelle du laboratoire.

paille - traitement - complémentation - énergie - protéines - génisses - croissance hiver 
Summary - Respective value of ammonia treatment and appropriate supplementation of wheat straw (level and nature of supplementary energy and nitrogen) in two year old winterIng dairy heifers. The aim of this study was to : (1) better evaluate the minimum energy supplementation level of correctly $\mathrm{NH}_{3}$-treated wheat straw, (2) check the interest of a supply of low and slowly rumen degradable proteins, (3) evaluate whether or not an appropriate supplementation of straw would allow either a better response of the treatment or even replace that treatment itself.

We carried out 3 trials (I, II, III) respectively during the 1983-84, 1984-85 and 1985-86 winter periods on 5, 6 and 4 groups (11 animals per group) of dairy heifers (2 years old, FFPN breed) moderately growing $(350 \mathrm{~g} / \mathrm{j})$, fed wheat straw (var. Hardy), all originating from the same field. This straw was fed ad libitum (10-15\% refusal) either untreated (US) or ammonia-treated (TS) : by the FMA oven technique ( $30 \mathrm{~g} \mathrm{NH}$ per $\mathrm{kg}$ straw) in trial lor by the polyethylene - stack technique $(50 \mathrm{~g}$ $\mathrm{NH}_{3}$ per $\mathrm{kg}$ straw) in the 3 trials. The levels and the nature of the supplements used in each trial are given in Table 1. Both US and TS were supplemented with $1.5 \mathrm{~kg} \mathrm{DM}$ of a mix-pasture good quality silage and with dehydrated sugar beet pulp. $2.6 \mathrm{~kg} / \mathrm{d}$ of DM beet pulp were fed in addition to US and, either the same quantity, or intermediary quantities $(2.00$ and $0.90 \mathrm{~kg})$, or no $(0 \mathrm{~kg})$ beet pulp $D M$, were fed in addition to TS (Table I). $90 \mathrm{~g}$ urea was added to the US which provided an amount of NPN similar to that supplied by the treatment $\mathrm{NH}_{3}$. Nitrogen (in terms of digestible proteins in the intestine, french PDI) supplementation levels, which are identical from one group to another within a given trial, were calculated from the theoretical $N$ requirements for the $350 \mathrm{~g} / d$ liveweight gain (LWG) target in such a way that the final CP content of the diet DM was $12 \%$. Taned soybean oilcake, which permitted supply of low degradable N (of dietary origin, ie french PDIA), was supplemented in excess as compared with the above theoretical $N$ requirements, to some of the groups: $0.40 \mathrm{~kg} / \mathrm{d}$ for US and $0.25 \mathrm{~kg} / \mathrm{d}$ for TS. Heifers were given $120 \mathrm{~g} / \mathrm{d}$ of a $10 / 18 \mathrm{mineral}$ mixture.

In addition to the classical analysis performed on straw and supplements (Table II), we measured the in vivo organic matter digestibilities $(O M D)$, at maintenance level, and the voluntary DM intakes of the various straws with groups of sheep.

Experimental phases of trials carried out with heifers are indicated in Figures 1,2 and 3. They lasted 71,98 and 77 days respectively for trials I, II and III. Each of them were limited by both 2 to 3-week pre- and post-experimental periods during which all groups were fed the same diet. We thus could correct the liveweights from the digestive tract content weight, which was considered to average $6 \mathrm{~kg}$ DM per each $\mathrm{kg} D \mathrm{DM}$ of straw ingested.

OMD and voluntary intake of various straws by sheep are given in Table II. OMD of the 3 US (39.4 - 35.9 and $34.8 \%$ ) were improved by $\mathrm{NH}_{3}$ treatments respectively to 52.9 (oven) and $52.7 \%$ (stack) in trial I and to 50.9 and $51.6 \%$ in trials II and III. US DM intakes, which were rather low, 17.0 -26.0 and $23.1 \mathrm{~g} / \mathrm{kg} \mathrm{L.W.} 0.75$, were improved to the respective values of 29.3 (oven) and 34.3 (stack) in trial I and of 29.1 and $30.1 \mathrm{~g} / \mathrm{kg} \mathrm{L.W.0.75}$ in trial II and III. Improvements of these values, as well as the CP content of TS, reveals that all treatments were perfectly satisfactory.

Average straw intakes and daily gains with heifers are given in Table III. Figures 1, 2 and 3 show, in addition, the variation of these values during the various trials.

US DM intakes with heifers, very low in trial / $(2.81 \mathrm{~kg} / \mathrm{d})$, were highest in trial $/ 1(4.36 \mathrm{~kg} / \mathrm{j}) . \mathrm{DM}$ intakes were improved with treatments but to a very variable extent, depending upon the proportion of pulp in the rations. The percentages of improvement ranged from $11-28$ and $67 \%$ with high pulp levels $(2.6 \mathrm{~kg} / \mathrm{d}$, the same as with US, and $2.0 \mathrm{~kg} / \mathrm{d})$, to 27 and $80 \%$ with low quantities of pulp (around $1.0 \mathrm{~kg} / \mathrm{d}$ ) and to $87 \%$ when TS were fed without any pulp.

All average daily live-weight gains (ADG) were equal or higher to those expected with the only exception of US in trial I (Table V). Ammonia treatments therefore allowed the ADG to increase (significantly) from 218 to 550 and to $441 \mathrm{~g} / \mathrm{d}$ in trial I where supplementations of both US and TS were the same. However, the treatment did not permit reducing the totality of the pulp supplementation without reducing $A D G$ (nil in trial l), despite, in that case, the improvement of the energy value of straw (+ 0.30 UFL) and of straw intake. For the same given ADG between US and TS, the ammonia treatment, when correctly achieved, allows reduction of the beet pulp supplement to an order of 1.8 to $1.9 \mathrm{~kg}$ DM per day (which represents roughly a saving of 1.5 UFL per day). The improvement of the energy value of TS, which then represents 65 to $70 \%$ of the rations, is only of the order of 0.2 UFL $/ \mathrm{kg}$ DM. These results are in accordance with those of some authors which show, in animals, with moderate requirements the importance of both the nature and the level of the energy supplement with regard to optimizing the treatment benefit. 
The supply of a low degradable $N$ source (PDIA), in excess with regard to actual theoretical requirements, did not show any marked improvement of either US or TS DM intakes. However, it could permit increasing (non significantly, but still interesting) the ADG of heifers in both cases of US and TS (Table III). ADG of US fed heifers could even reach the same value as TS heifers (with or without oil cakes). One may therefore wonder, in these conditions, whether the treatment is still necessary. The answer to such a question does resort to other agro-economical considerations that should be thoroughly analysed. In addition, when positive, the response on ADG of supplying low and slowly degradable $\mathrm{N}$ confirms and gives more evidence to observations already obtained (but only at the laboratory level until now) by some other authors and ourselves, when feeding either protected oil-cakes or fish meal. As a matter of fact it was shown that such a protein-rich supplementation was beneficial to the «host" animal but also to the cellulolytic rumen microorganisms activity. The latter take advantage of amino-acids supply. They also benefit, in their synthesis and activity, of a better synchronisation of the release of both energy and $\mathrm{NH}_{3}$, then more efficient than with classical NPN sources.

straw - treatment — supplementation - energy - protein - heifers - growth - winter

\section{INTRODUCTION}

L'Europe connaît, depuis ces dernières années, un développement important du traitement de pailles. En effet, depuis la mise au point du traitement à l'ammoniac (sous bâches plastique) proposé par Sundstøl, Coxworth et Mowat (1978), l'éleveur peut améliorer la valeur de ses pailles de façon simple, moins coûteuse et moins dangereuse qu'avec la soude (Cordesse, 1987).

S'il existe déjà beaucoup de résultats d'essais d'alimentation d'animaux à besoins modérés recevant des pailles traitées à l'ammoniac $\left(\mathrm{P}^{\mathrm{NH}} \mathrm{N}_{3}\right.$ ), les travaux récents sur la valeur nutritive de ces pailles traitées montrent qu'on peut encore se poser des questions sur l'intérêt zootechnique de ce traitement (Demarquilly, Chenost et Ramihone, 1987).

Le traitement à l'ammoniac augmente en effet la digestibilité et l'ingestibilité des pailles, et cela d'autant plus que les valeurs correspondantes de la paille non traitée sont faibles (Tuah et al., 1986; Chenost \& Demarquilly, 1985). Toutefois quelques auteurs (Dulphy et al., 1984; Ramihone \& Chenost, 1987) ont montré que, toutes choses égales par ailleurs, les régimes à base de $\mathrm{P} . \mathrm{NH}_{3}$ entraînaient par rapport à ceux à base de pailles non traitées une diminution de l'activité cellulolytique du jus de rumen. En outre, pour Demarquilly et al. (1987) l'équivalent de 24 à $37 \%$ de l'azote “fixé » grâce au traitement n'est pas utilisé et se retrouve dans les fèces. La teneur en matières azotées non digestibles des pailles passe ainsi de $38 \mathrm{~g} / \mathrm{kg}$ MS avant traitement à $56-57 \mathrm{~g}$ après traitement.

Par ailleurs, les résultats des essais français, résumés par Demarquilly et al. (1987) montrent que l'augmentation de la valeur énergétique, entraînée par le traitement et calculée à partir des croissances de génisses recevant la paille traitée à volonté et la même complémentation qu'avec la paille non traitée, est nettement inférieure $(+0,02 \mathrm{UFL} / \mathrm{kg}$ de MS en moyenne) à celle estimée à partir des mesures de digestibilité sur moutons (+ 0,15 UFL en moyenne). Ceci peut être lié à la quantité, à la nature et aux modalités de l'apport du complément "supplémentaire", dont l'animal hôte a besoin pour couvrir ses besoins propres d'entretien et de production (Chenost, 1987; Chenost et al., 1987). On sait en effet (Dulphy et al., 1984) qu'un complément énergétique riche en parois aisément digestibles facili- 
tant la cellulolyse est préférable à de l'amidon et qu'il convient de le fractionner en plusieurs repas.

Les trois essais rapportés ici avaient pour but de : 1) compléter les observations de Dulphy et al. (1984a) et de Dulphy et al. (1984b) sur le niveau minimum de la complémentation énergétique suivant le type de traitement à l'ammoniac pour assurer à des génisses laitières de 2 ans des croissances hivernales de 350 g/jour; 2) vérifier si un apport supplémentaire d'azote, en particulier de protéines peu fermentescibles (tourteau tanné), améliorait les performances des animaux en compensant la mauvaise utilisation de l'azote fixé lors du traitement et, comme l'avaient suggéré certains auteurs (Preston \& Leng, 1984; Chesson \& Ørskov, 1984), en améliorant leur appétit et leur état nutritionnel; 3) voir dans quelle mesure une telle complémentation permettrait de mieux valoriser le traitement, voire même, de le remplacer.

\section{MATÉRIEL ET MÉTHODES}

\section{Dispositif expérimental}

\section{Pailles}

Les pailles utilisées au cours des trois essais successifs provenaient d'un blé d'hiver de variété Hardy cultivé dans la même exploitation située en Limagne. Elles étaient toutes conditionnées en balles cubiques de $13 \mathrm{~kg}$ (moyenne densité). Acheminées au Centre de Theix, peu de temps après leur récolte, elles étaient alors immédiatement, pour une partie, stockées en l'état sous hangar et, pour une autre partie, traitées à l'ammoniac anhydre (dans le cas des traitements en meules).

La paille de l'essai | (1983-1984) a été récoltée en août dans de bonnes conditions cli- matiques après une maturation de blé normale. Elle a été traitée soit en plusieurs "tas". (de 5 tonnes chacun) recouverts d'une bâche plastique, selon la technique proposée par Sundstøl et al. (1978), à raison de $50 \mathrm{~g} \mathrm{~d}^{\prime} \mathrm{NH}_{3}$ par $\mathrm{kg}$ de paille brute, soit en "four" FMA (cycle de $15 \mathrm{~h}$ à $90^{\circ} \mathrm{C}$, à raison de $30 \mathrm{~g} \mathrm{~d}^{\prime} \mathrm{NH}_{3} / \mathrm{kg}$ de paille). Le traitement en four, d'une capacité de 2 tonnes, était effectué 3 fois par semaine tout au long de l'essai.

Les pailles des essais II et III n'ont été traitées qu'en tas, à raison de $50 \mathrm{~g} \mathrm{~d} \mathrm{NH}_{3}$ par $\mathrm{kg}$ de paille. La paille de l'essai II avait reçu un peu de pluie avant le ramassage, la maturation du blé avait été normale. La paille de l'essai III, récoltée dans de bonnes conditions climatiques, contenait beaucoup d'adventices et provenait d'une culture ayant subi 1 mois de maturation particulièrement sec.

\section{Animaux}

On a utilisé, pour les trois essais, des génisses de race française Frisonne Pie Noire, âgées d'environ 2 ans, qui pesaient en moyenne 465 $\mathrm{kg}$ en début d'expérience pour les essais I et II et de 475 à $480 \mathrm{~kg}$ pour l'essai III. Les génisses étaient maintenues en stabulation libre (alimentation en lot) avec auvent, sur aire paillée, par cases équipées soit de 5 , soit de 6 cornadis. Chaque lot expérimental, composé de 11 génisses (couplage de deux cases de 5 et 6 places), a été constitué de façon que l'âge et le poids moyens des animaux soient comparables d'un lot à l'autre dans un essai donné. Le renouvellement de la litière, tout en restant suffisant, était effectué suivant une fréquence telle que les génisses en consomment le moins possible.

\section{Régimes expérimentaux}

Dans les trois essais, les pailles, traitées ou non, étaient distribuées à volonté (10 à 15\% de refus) sous forme longue, en 2 repas par jour. Elles ont été systématiquement complémentées avec 1,3 à $1,4 \mathrm{~kg}$ de MS d'un ensilage d'herbe de bonne qualité (1er cycle d'une prairie naturelle réalisé par coupe fine avec conservateur). Les autres compléments, pulpes de betteraves déshydratées et tourteau de soja tanné, ont été apportés selon des quantités variant avec les objectifs expérimentaux que nous avons résumés dans le tableau I. Ensilages, pulpes et tourteaux étaient également apportés en 2 repas par jour, une demi-heure à 
une heure avant la distribution de la paille. On rajoutait $90 \mathrm{~g}$ d'urée par jour aux rations des génisses consommant les P.NT (en deux apports par jour, en même temps que les aliments complémentaires); cette quantité d'azote non protéique correspondait, en moyenne, à la quantité d'azote fixée sur les pailles après leur traitement à l'ammoniac. L'adjonction d'urée n'a pas tenu compte du fait que $30 \%$ de cet azote fixé n'était pas utilisé.

L'essai I a été effectué sur 5 lots d'animaux qui recevaient : la paille non traitée (P.NT), lot

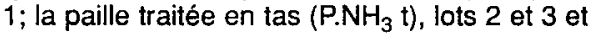
la paille traitée au four ( $P . \mathrm{NH}_{3} f$ ), lots 4 et 5 . La complémentation en pulpe a été de $2,6 \mathrm{~kg}$ de MS par jour pour le lot P.NT et pour deux des lots P.NH $\mathrm{NH}_{3}$, elle a été nulle pour les 2 autres lots $\mathrm{P.NH}_{3}$, (Tableau I).

Les essais II et llI ont été effectués respectivement sur 6 et 4 lots. La complémentation en pulpe des lots P.NT était la même que pour le lot P.NT de l'essai I. Elle a été ramenée, pour les lots $\mathrm{P}_{\mathrm{NH}} \mathrm{N}$, à une valeur de l'ordre de 2 $\mathrm{kg} / \mathrm{j}$ pour les lots 3 et 4 de l'essai II et de $1 \mathrm{~kg} / \mathrm{j}$ pour les lots 5 et 6 de l'essai II et 3 et 4 de l'essai III. Du tourteau de soja tanné a été distribué à un lot sur deux dans chacun de ces 2 essais à raison de $0,4 \mathrm{~kg} / \mathrm{j}$ pour les P.NT et de 0,2 à $0,3 \mathrm{~kg} / \mathrm{j}$ pour les $\mathrm{P} . \mathrm{NH}_{3} \mathrm{t}$, (Tableau I).
Indépendamment de la nature des apports azotés la teneur des rations en équivalent $\mathbf{N} \times$ 6,25 était de 10 à $12 \%$ de la MS pour chacun des lots des 3 essais. Les quantités totales de PDI apportées à chaque lot étaient, en outre, au moins égales (supérieures dans la plupart des cas) à celles théoriquement nécessaires (INRA, 1988) pour les croissances visées $(350 \mathrm{~g} / \mathrm{l})$. Nous pouvions ainsi vérifier l'intérêt d'un apport de protéines peu dégradables (source de PDIA) sous forme de tourteau tanné, l'une des hypothèses formulée plus haut. Pour un même apport de PDI entre lots recevant ou non le tourteau tanné (510 à $640 \mathrm{~g}$ de PDIN et 528 à $627 \mathrm{~g}$ de PDIE, pour des besoins théoriques variant de 422 a $500 \mathrm{~g}$ de PDI, suivant les paires de lots) la part des PDIA a été de 60 à $115 \mathrm{~g}$ plus élevée chez les lots recevant le tourteau tanné que chez ceux n'en recevant pas.

Dans chaque essai les animaux recevaient $100 \mathrm{~g} / \mathrm{j}$ d'un complément minéral vitaminé (10 $P, 18 \mathrm{Ca}$ ) enrichi en oligo-éléments, dont la compostion est donnée au tableau I, plus $20 \mathrm{~g} / \mathrm{J}$ d'un mélange de fleur de soufre et de $\mathrm{SO}_{4} \mathrm{Na}_{2}$. Une injection de vitamines A1, D3 et E était en outre administrée tous les mois à chaque génisse.

\section{Essai I - Trial I}

(a)

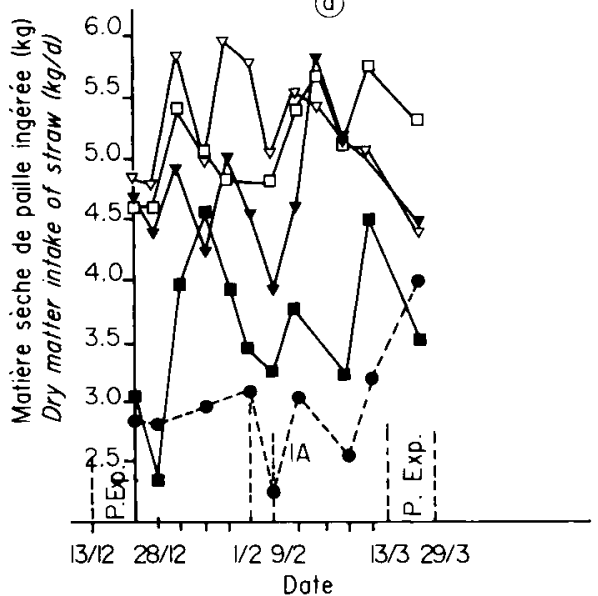

(b)

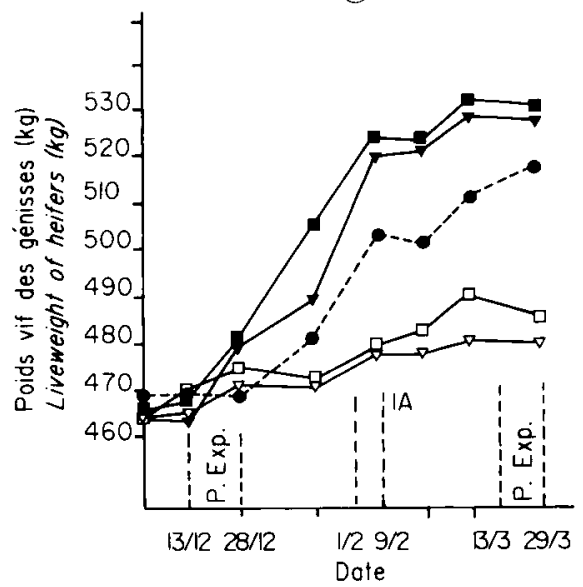

Fig. 1. Variation (a) des quantités moyennes journalières de matière sèche de pailles ingérées et (b) du poids vif moyen des génisses suivant les lots expérimentaux au cours de l'essai I (1983-1984). lot $1:$ PNT + urée; $\square$ lot $2: \mathrm{PNH}_{3}$ t sans pulpe (op); lot $3: \mathrm{PNH}_{3} t+$ pulpe (+ p); $\nabla$ lot $4: \mathrm{PNH}_{3} f$ $+(\mathrm{op}) ; \nabla$ lot $5: \mathrm{PNH}_{3} \mathrm{f}+\mathrm{p} ; \mathrm{IA}$ : insémination artificielle; $p$. Exp : période pré-expérimentale; $P$. Exp. : période post-expérimentale. 
M. Chenost

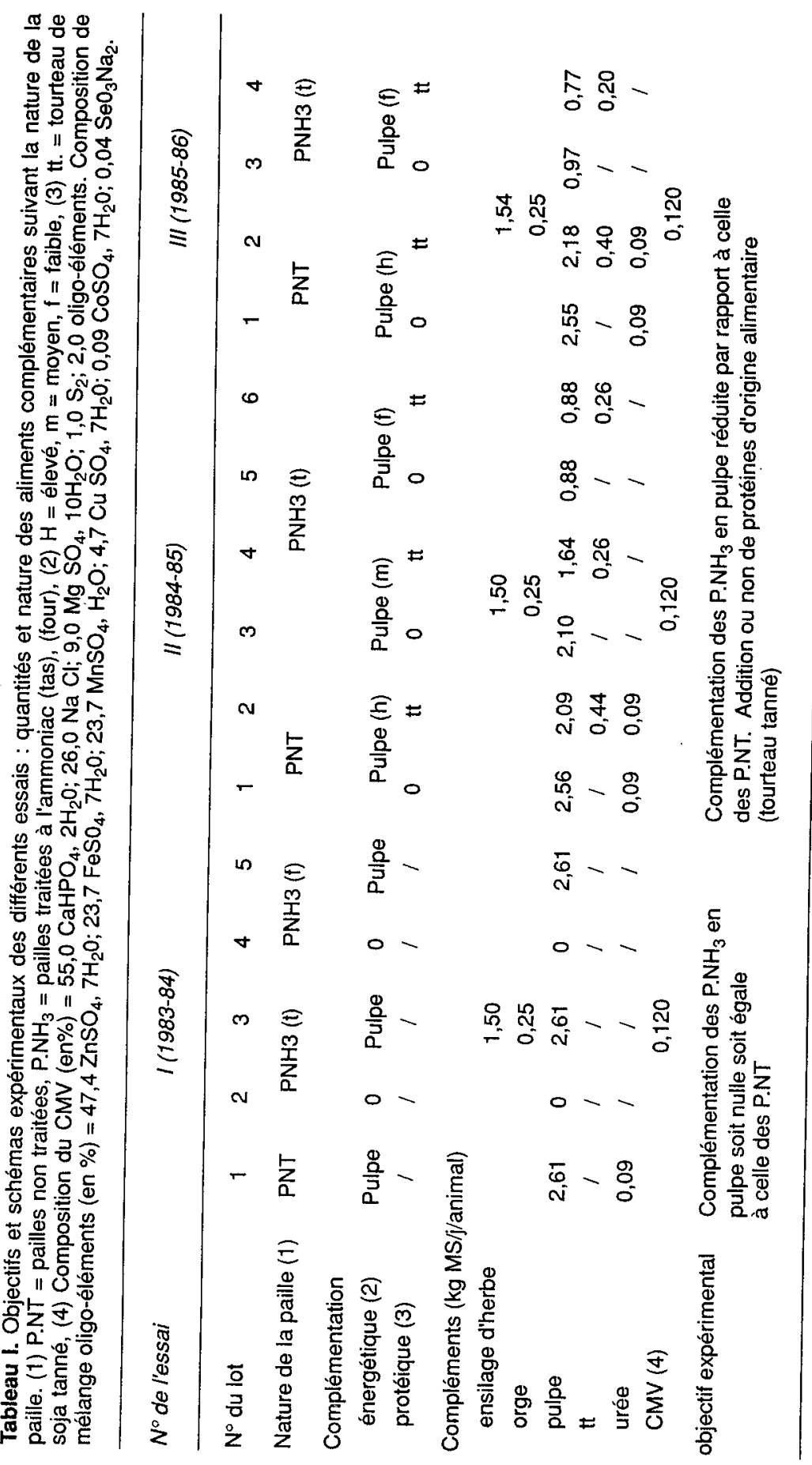



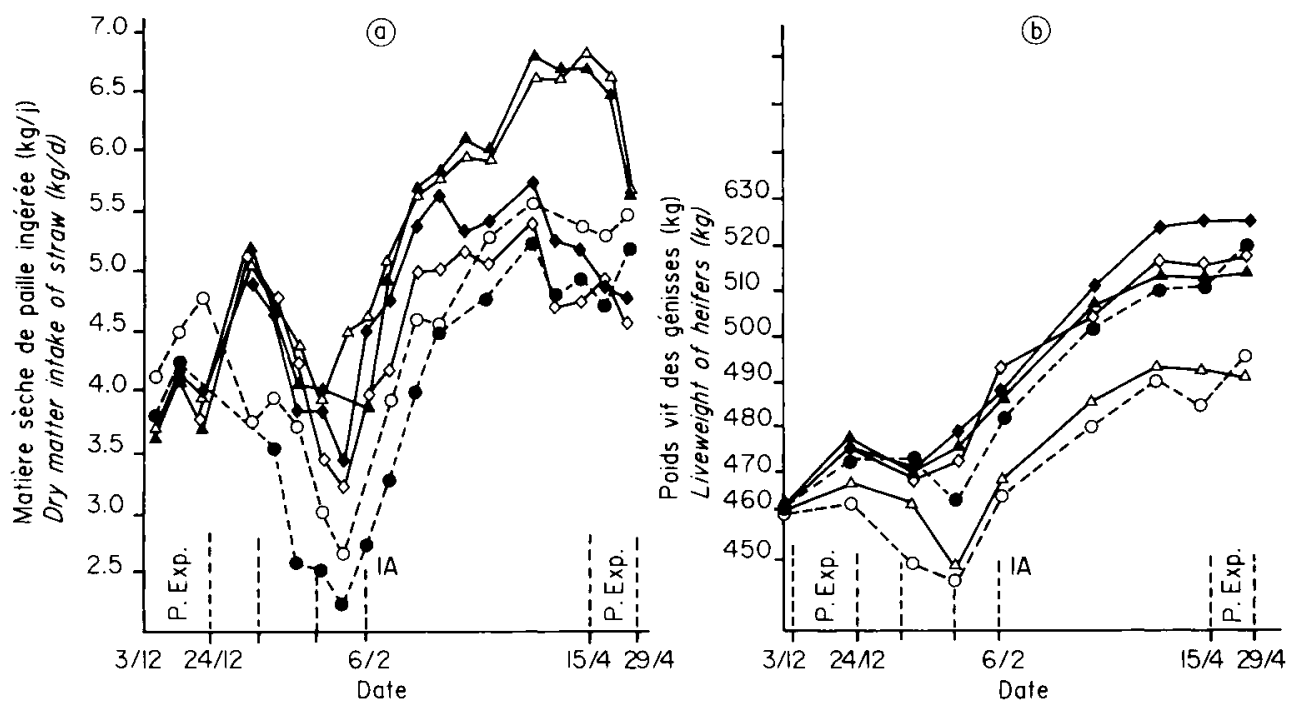

Fig. 2. Variations (a) des quantités moyennes journalières de matière sèche de pailles ingérées et (b) du poids vif moyen des génisses suivant les lots expérimentaux au cours de l'essai II (19841985). O lot $1:$ PNT + urée; lot $2:$ PNT + tourteau tanné (tt); $\diamond$ lot $3: P^{2} T_{3} t+p 2+0 ;-$ lot 4 : $\mathrm{PNH}_{3} \mathrm{t}+\mathrm{p} 2+\mathrm{t} ; \Delta$ lot $5: \mathrm{PNH}_{3} \mathrm{t}+\mathrm{p} 1+0 ; \Delta$ lot $6: \mathrm{PNH}_{3} \mathrm{t}+\mathrm{p} 1+\mathrm{tt}$. $\mathrm{p} 2=$ niveau élevé de pulpe; $\mathrm{p} 1=$ niveau bas de pulpe.

\section{Essai III - Trial III}
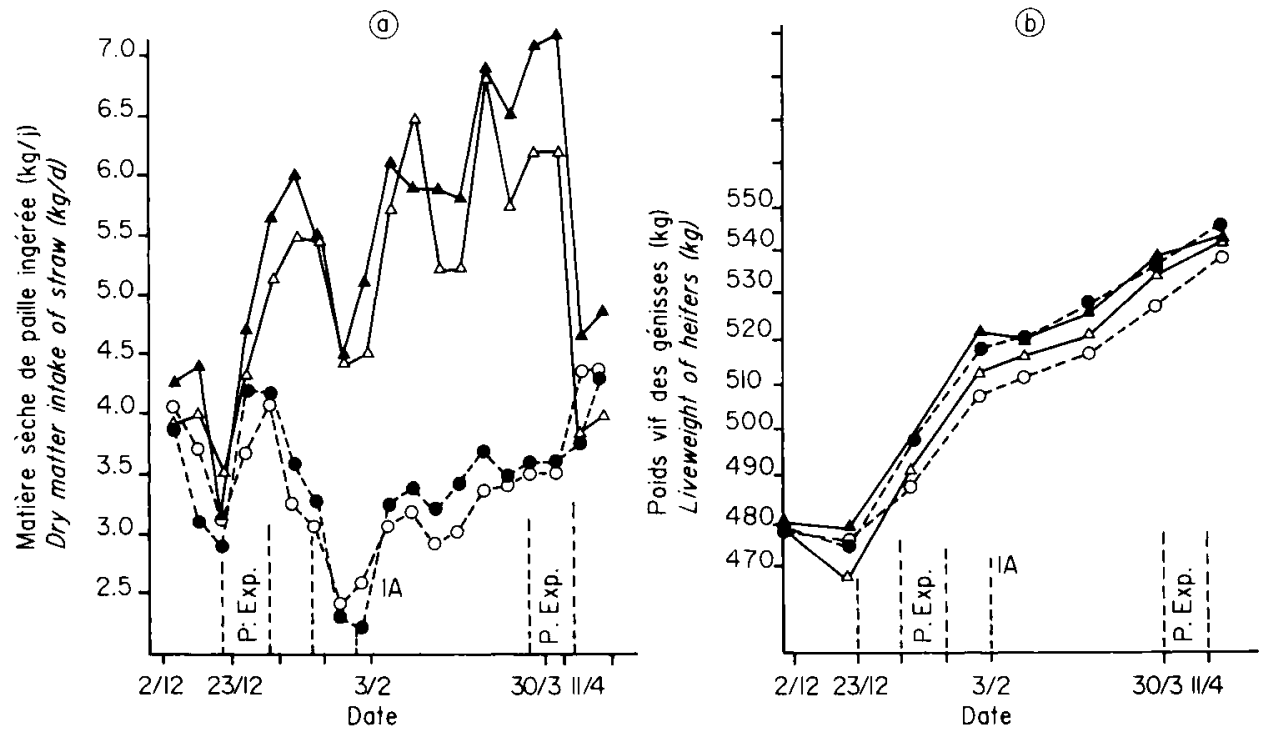

Fig. 3. Variations (a) des quantités moyennes journalières de matière sèche de pailles ingérées et (b) du poids vif moyen des génisses suivant les lots expérimentaux au cours de l'essai III (19851986). $\mathrm{O}$ lot $1: \mathrm{PNT}+\mathrm{p} 2+0 ; \bigcirc \operatorname{lot} 2: \mathrm{PNT}+\mathrm{p} 2+\mathrm{tt} ; \Delta$ lot $3: \mathrm{PNH}_{3}+\mathrm{p} 1+0 ; \Delta \operatorname{lot} 4: \mathrm{PNH}_{3}+\mathrm{p} 1$ + tt. Mêmes légendes que sur les figures 1 et 2 . 


\section{Déroulement des essais}

Les calendriers de déroulement des trois essais apparaissent sur les figures 1, 2 et 3.

Les périodes expérimentales $(71,98,77$ jours, respectivement pour les essais I, II et III) étaient toujours encadrées de périodes pré- et post-expérimentales (de 2 à 3 semaines chacune) pendant lesquelles tous les lots recevaient le même régime (pailles non traitées) de façon à pouvoir corriger les poids vifs de la variation du poids des contenus digestifs dus aux régimes expérimentaux. On a pris pour cela comme hypothèse qu'une variation d'ingestion de $1 \mathrm{~kg}$ de matière sèche de paille entraînait une variation du poids du contenu digestif, donc de poids vif, de $6 \mathrm{~kg}$. Une période de transition de l'ordre de 2 semaines suivait la période expérimentale proprement dite afin de substituer progressivement le régime expérimental au régime pré-expérimental.

Les génisses ont été inséminées à des dates tombant pendant les périodes expérimentales (Figs. 1, 2 et 3 ). Une période de flushing d'environ 10 jours pendant laquelle des génisses recevaient $1,7 \mathrm{~kg}$ de MS d'orge supplémentaire par jour précédait l'insémination artificielle.

\section{Mesures}

Les génisses ont été pesées tous les 15 jours (pesées simples), et au début et à la fin de chaque période intermédiaire (double pesée).

Les quantités de matière sèche de paille ingérées ont été mesurées 3 jours par semaine pendant toute la durée des essais. Un prélèvement effectué à cette occasion a permis de constituer un échantillon cumulé représentatif des pailles ingérées pendant les périodes expérimentales. Une fraction des échantillons de pailles traitées n'était séchée qu'à $40^{\circ} \mathrm{C}$ en vue de l'analyse des diverses fractions azotées; l'autre fraction était séchée à $80^{\circ} \mathrm{C}$ pour la mesure de la teneur en matière sèche et pour les autres dosages.

Un échantillon représentatif des pailles, des ensilages, des pulpes déshydratées et du tourteau de soja tanné a été constitué pour chaque essai. Les Tableaux lla et b donnent les résultats des dosages effectués sur chaque constituant des régimes expérimentaux.

Pour chaque essai on a également mesuré, sur moutons, la digestibilité (en quantité limitée à $30 \mathrm{~g} \mathrm{MS} \mathrm{kg}^{-1} \mathrm{P}^{-0,75} \mathrm{j}^{-1}$ et les quantités volontairement ingérées (distribution ad libitum, 10 à $15 \%$ de refus) des pailles non traitées et des pailles traitées. La digestibilité des pailles non traitées a été calculée par différence entre celle de la ration totale (comportant paille plus $350 \mathrm{~g}$ d'un mélange $50 / 50$ de maïs et de tourteau de soja) et celle des concentrés, données par les tables INRA (1988). Les pailles traitées ayant une teneur en $\mathrm{N} \times 6,25$ de $10 \%$ n'ont pas été complémentées, leur digestibilité a donc été obtenue directement.

Nous avons estimé la valeur énergétique des pailles à partir, soit des besoins théoriques correspondant aux croissances réalisées par les génisses en affectant aux compléments utilisés les valeurs UFL données dans les tables INRA (1988), soit à partir des digestibilités de la matière organique mesurées sur moutons. L'analyse statistique des croîts a été effectuée avec le test de Duncan (1955).

\section{RÉSULTATS}

Les périodes de référence servant à calculer les quantités moyennes de matière sèche ingérées et les croîts journaliers moyens correspondent habituellement aux périodes expérimentales. Or, au cours des essais I et II les consommations de paille ont chuté pendant la période pré-expérimentale (pourtant progressive) et, même, pendant la période de transition (substitution progressive des régimes expérimentaux au régime préexpérimental). Les poids initiaux des périodes expérimentales étaient, par conséquent, plus faibles que ce qu'ils auraient dû être, et très variables d'un lot à l'autre; les croîts ont ainsi été surestimés artificiellement, notamment dans l'essai II. Nous avons donc préféré prendre en compte les périodes totales (pré- et post-expérimentales en plus des périodes expérimentales) pour le calcul des croîts moyens de ces 2 essais. 
Tableau lla. Composition chimique et valeur alimentaire des pailles suivant les essais.

\begin{tabular}{|c|c|c|c|c|c|c|c|}
\hline \multirow{2}{*}{$\begin{array}{l}\text { Essais } \\
\text { Pailles (1) }\end{array}$} & \multicolumn{3}{|c|}{ । } & \multicolumn{2}{|c|}{ ॥ } & \multicolumn{2}{|c|}{ III } \\
\hline & P.NT & P.NH $H_{3} t$ & $\mathrm{P} \mathrm{NH}_{3} \mathrm{f}$ & P.NT & P.NH ${ }_{3} \mathrm{t}$ & P.NT & P.NH $H_{3} \mathrm{t}$ \\
\hline \multicolumn{8}{|l|}{ Composition \% } \\
\hline Cendres & 10,4 & 8,8 & 9,8 & 9,9 & 6,4 & 8,0 & 9,5 \\
\hline$N \times 6,25$ & 2,9 & 9,4 & 10,1 & 3,9 & 10,3 & 4,5 & 11,1 \\
\hline $\mathrm{N}$ soluble $(\%)$ & & 57 & 48 & & 46 & & 67 \\
\hline \multicolumn{8}{|l|}{$\mathrm{N}$ total } \\
\hline $\mathrm{N}$ fixé (\% de $\mathrm{N}$ injecté) & 一 & 25,3 & 46,8 & - & 24,9 & - & 25,7 \\
\hline Cellulose brute & & 44,4 & 44,5 & 38,5 & 42,5 & 39,8 & 39,8 \\
\hline NDF & & 73,3 & 74,1 & 77,7 & 80,0 & 69,0 & 74,7 \\
\hline ADF Van Soest & & 49,9 & 50,3 & 53,3 & 54,4 & 43,4 & 50,9 \\
\hline \multicolumn{8}{|l|}{ Valeur alimentaire (mouton) } \\
\hline dmo $(\%)$ & 39,4 & 52,7 & 52,9 & 35,9 & 50,9 & 34,8 & 51,6 \\
\hline MS ingérée Ad. lib. (g/kg P0.75) & 17,0 & 34,3 & 29,3 & 26,0 & 29,1 & 23,1 & 30,1 \\
\hline Valeur énergétique nette (UFL/kg MS) & 0,36 & 0,54 & 0,53 & 0,39 & 0,53 & 0,34 & 0,55 \\
\hline
\end{tabular}

Tableau Ilb. Composition et valeur alimentaire des compléments suivant les essais. (1) Mêmes abréviations que le Tableau I; (2) Unité fourragère pour la production de lait d'après les tables INRA 1988.

\begin{tabular}{|c|c|c|c|c|c|}
\hline \multirow[t]{2}{*}{ Essais } & \multirow[t]{2}{*}{1} & \multirow{2}{*}{$\begin{array}{c}\| \\
\text { ensilages }\end{array}$} & \multirow[t]{2}{*}{ III } & \multicolumn{2}{|c|}{ Moyenne des 3 essais } \\
\hline & & & & pulpe & tourteau tanné \\
\hline MS (\%) & 18,0 & 17,2 & & & \\
\hline $\mathrm{pH}$ & 4,72 & 4,23 & & & \\
\hline MAT (\% MS) & 15,9 & 13,4 & 17,0 & 9,5 & 45,4 \\
\hline $\mathrm{N}$ soluble (\% $\mathrm{N}$ total) & 58,8 & 57,0 & 50,6 & & \\
\hline Acide lactique (\% MS) & 10,9 & 5,4 & & & \\
\hline UFL/kg MS & & 5 à 0,90 & & $0,90(2)$ & $1,20(2)$ \\
\hline
\end{tabular}


Aussi ne donnons-nous que les croîts calculés pendant les périodes expérimentales pour les essais II et III et ceux calculés pendant toute la durée de l'expérience pour l'essai I, à titre de comparaison. En revanche nous n'avons considéré que les périodes expérimentales pour le calcul des quantités moyennes de matière sèche ingérées dans les 3 essais.

\section{Valeur nutritive des pailles et effets de la nature du traitement à l'ammoniac}

La digestibilité et surtout l'ingestibilité des trois pailles utilisées ont été moyennes à médiocres (Tableau lla) et inférieures aux valeurs moyennes des pailles de blé étudiées dans notre laboratoire (Andrieu \& Demarquilly, 1987). La paille de l'essai I a été très mal ingérée $\left(17 \mathrm{~g} / \mathrm{kg} P^{0,75}\right)$ malgré sa digestibilité plus élevée que celles des pailles des essais II et III $(39,4$ contre 35,9 et $34,8 \%$ ). Sa teneur en MAT $(2,9$ contre 3,9 et 4,5 ) était très faible.

Les traitements à l'ammoniac ont permis de faire passer la teneur en équivalent matières azotées $(N \times 6,25)$ des pailles à des valeurs comprises entre 9,4 et $11,1 \% \mathrm{MS}$, valeurs tout à fait correctes et comparables à celles observées généralement dans la littérature et dans la pratique lorsque les traitements sont réussis. Le traitement au four, grâce à l'apport de chaleur, permet par rapport au traitement en tas, non seulement une économie d'ammoniac (30 contre $50 \mathrm{~g} / \mathrm{kg}$ de MS de paille) mais également une augmentation du pourcentage d'azote retenu par rapport à l'azote utilisé pour le traitement (de l'ordre de 47 contre 25, Tableau Ila).

Indépendamment de leur nature, les traitements ont permis de faire passer la digestibilité de la matière organique des pailles à des valeurs correctes et voisines pour les trois pailles (de 50,9 à 52,9\%). Celles-ci correspondent à des augmentations de digestibilité comprises entre 13,3 et 16,8 points qui reflètent, là aussi, la bonne réussite des traitements.

L'augmentation des quantités de paille ingérées, également de l'ordre de celle observée dans le cas de traitements réussis, a été en faveur du traitement en tas avec les moutons (Tableau Ila) et du trajtement au four avec les génisses (du moins lorsque leur complémentation en pulpe était réduite, Tableau III). Dans ce cas, toutefois, l'augmentation des quantités ingérées de paille traitées au four s'est traduite par une diminution, non significative, du croît des génisses (Tableau III).

\section{Niveau de la complémentation énergé- tique des pailles traitées. Essai I}

\section{Quantités de pailles ingérées}

Les consommations de pailles ont présenté des fluctuations hebdomadaires importantes et irrégulières pendant toute la période expérimentale. Elles ont surtout diminué, pour tous les lots, pendant la période de flushing où les animaux recevaient $2 \mathrm{~kg}$ d'orge en plus (Fig. 1a).

Les quantités moyennes de P.NT ingérées ont été de $2,81 \mathrm{~kg}$ de $\mathrm{MS} / \mathrm{j}$, soit 0,54 $\mathrm{kg} / 100 \mathrm{~kg}$ P.V. Ces chiffres, très faibles, confèrent à cette paille une valeur d'encombrement de 1,82 UEB (INRA, 1988) assez élevée (Tableau III).

Les quantités moyennes de pailles ingérées ont augmenté, respectivement, avec les traitements en tas et au four, de 28 et $67 \%$ lorsque les génisses recevaient la même quantité de pulpe que les "génisses P.NT" et de 82 et $87 \%$ lorsque les génisses recevaient les $\mathrm{P}_{\mathrm{NH}} \mathrm{NH}_{3}$ sans pulpes. Les valeurs d'encombrement des pailles traitées ont ainsi été ramenées à 1,43 et 1,10 (avec pulpes) et à 1,55 et 


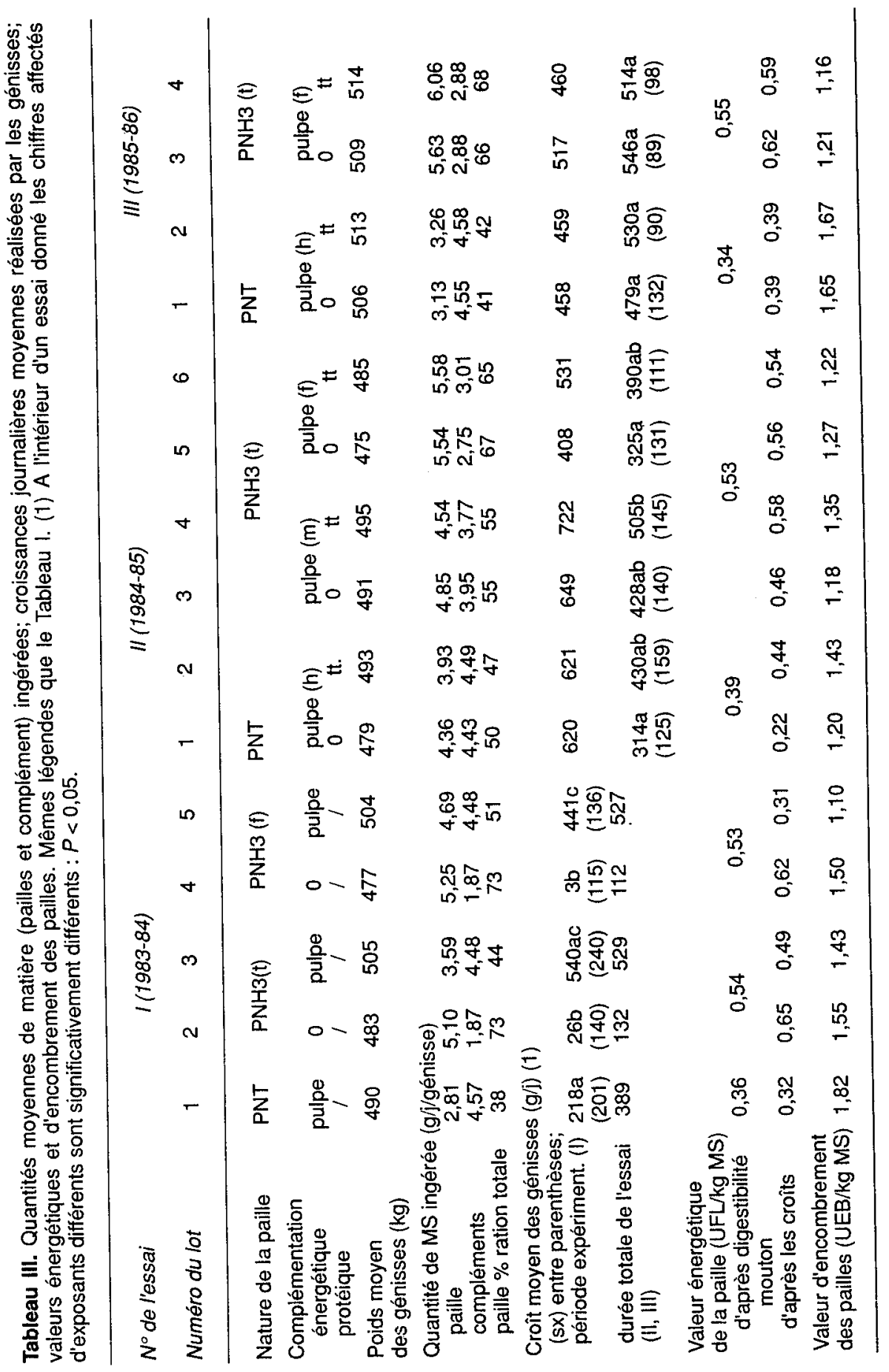


1,50 UEB (sans pulpes) respectivement pour les traitements tas et four. Les taux de substitution pulpe/paille ont été de 0,58 et 0,21 pour les pailles traitées en tas et au four.

Les pailles ont représenté respectivement $38-44$ et $51 \%$ (Tableau III) des rations P.NT, P.NH $\mathrm{NH}_{3}$ t et $\mathrm{P} . \mathrm{NH}_{3}$ f contenant des pulpes et 73 et $74 \%$ des rations P. $\mathrm{NH}_{3}$ t et P. $\mathrm{NH}_{3}$ f n'en contenant pas.

\section{Croîts journaliers}

Les croits journaliers moyens réalisés par les différents lots (Tableau III), reflètent bien l'évolution des poids reportés à la figure 1b; ils présentent toutefois une grande variabilité ( $\mathrm{Sx}$ est compris entre 115 et $240 \mathrm{~g} / \mathrm{j}$ ).

Les croîts obtenus avec les pailles non traitées (Tableau III) n'ont été que de 218 $\pm 201 \mathrm{~g} / \mathrm{j}$, et ceux obtenus avec les pailles traitées sans pulpe pratiquement nuls (Tableau III). Seuls les croîts réalisés avec les pailles traitées complémentées par la pulpe $(540 \pm 240$ et $441 \pm 136 \mathrm{~g} / \mathrm{j}$ respectivement pour les pailles meules et four, non différents entre eux) ont été significativement $(P<0,05)$ supérieurs à ceux du lot paille NT et ont atteint ou dépassé les objectifs fixés, $350 \mathrm{~g} / \mathrm{j}$. A complémentation égale entre P.NT et P. $\mathrm{NH}_{3}$ (2,6 kg de MS de pulpe) les traitements tas et four ont ainsi permis d'augmenter les croissances respectivement de 322 et de $223 \mathrm{~g} / \mathrm{j}$ (lots 3 et 5 ).

\section{Niveau optimum de la complémenta- tion énergétique et nature de la com- plémentation azotée des pailles trai- tées. Essais II et III}

\section{Quantités de paille ingérées}

Les consommations de pailles observées pendant les essais II et III ont augmenté tout au long des périodes expérimentales (Figs. 2a et 3a), mais de manière fluctuante par suite des chutes de consommation momentanées dues aux périodes de froid (le mois de janvier dans ces 2 essais a été particulièreirient froid) et au flushing.

Les quantités moyennes de matière sèche des P.NT ingérées pendant l'essai II ont été de $4,36(0,98 \mathrm{~kg} / 100 \mathrm{~kg} P V)$ et de $3,93 \mathrm{~kg} / \mathrm{j}(0,84 \mathrm{~kg} / 100 \mathrm{~kg}$ PV) respectivement avec les complémentations urée et tourteau de soja (Tableau III). Elles ont été plus faibles dans l'essai III $(3,13$ et $3,26 \mathrm{~kg} / \mathrm{j})$, soit 0,65 et $0,61 \mathrm{~kg} / 100 \mathrm{~kg} P V$. Les valeurs d'encombrement calculées correspondantes sont ainsi de 1,20 (sans tourteau) et 1,43 UEB (avec tourteau) pour l'essai II et sont de l'ordre de 1,65 UEB, avec et sans tourteau dans l'essai III.

Les quantités moyennes de pailles ingérées ont augmenté avec le traitement, de $11 \%$ (essai II) avec la complémentation en pulpe élevée $(2,10 \mathrm{~kg} / \mathrm{j})$ et, respectivement, de 27 et $80 \%$ pour les lots des essais II et III ne recevant qu'une quantité réduite de pulpe $(0,88$ et $0,97 \mathrm{~kg} / \mathrm{j})$. L'effet bénéfique du traitement a été le plus net avec la paille de l'essai III dont la digestibilité et l'ingestibilité, mesurées sur moutons avant et après traitement, étaient pourtant comparables à celles de la paille de l'essai II. Les valeurs d'encombrement correspondantes, comprises entre 1,18 et 1,35 UEB pour l'essai II et de l'ordre de 1,2 UEB pour l'essai III, sont voisines de celles habituellement observées pour des pailles traitées à l'ammoniac.

L'adjonction de tourteau de soja tanné à la pulpe a eu un effet négatif $(3,13$ contre $4,36 \mathrm{~kg}$ MS ingérée par jour dans l'essai II) ou nul (3,13 contre 3,26 dans l'essai III) sur les quantités de P.NT ingérées. II n'a eu un effet positif sur les quantités de $\mathrm{P}_{\text {.NH }}$ ingérées que dans l'essai III (Tableau III). 
Les taux de substitution de la fraction pulpe plus tourteau à la paille traitée ne peuvent être calculés que dans l'essai II où les quantités de pulpe et de tourteau ont varié entre paires de lots $P . \mathrm{NH}_{3}$ (Tableau III). II est de 0,57 en l'absence de tourteau et de 1,37 avec le tourteau tanné.

\section{Croîts journaliers}

Les croîts journaliers moyens réalisés sont pratiquement tous supérieurs aux objectifs fixés. Seuls les croîts des lots 1 et 5 de l'essai II, respectivement P.NT sans tourteau et $\mathrm{P} . \mathrm{NH}_{3}$ sans tourteau et quantité réduite de pulpe, ont été légèrement inférieurs à $350 \mathrm{~g} / \mathrm{j}$ (314 et $325 \mathrm{~g} / \mathrm{j})$. Ces croîts moyens sont compris entre 314 et $546 \mathrm{~g} / \mathrm{j}$ et sont moins variables d'un lot à l'autre ou entre animaux pour un lot donné que dans l'essai l.

Les Figures $2 b$ et $3 b$, où nous avons reporté l'évolution des poids vifs moyens des différents lots, reflètent bien l'homogénéité de croissance des lots pendant l'essai II et, surtout, l'essai III. En effet, comme l'indique le Tableau III, seul le croît journalier réalisé pendant l'essai II

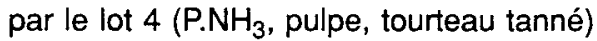
est significativement supérieur $(P>0,05)$ à ceux réalisés par les lots 1 et 5 (P.NT plus pulpe et P. $\mathrm{NH}_{3}$ plus pulpe). Les croîts réalisés pendant l'essai III ne sont pas significativement différents les uns des autres. Ils sont toutefois en moyenne plus élevés que pendant les essais I et II, très certainement en raison du poids plus élevé des génisses en début d'essai.

Comme la complémentation des P.NT et des P. $\mathrm{NH}_{3}$ n'a pas été la même selon les lots et les essais, l'effet bénéfique du traitement des pailles ne peut être jugé que sur ceux des lots ayant réalisé les mêmes croîts. L'effet bénéfique du traitement est alors mesuré en terme d'«économie" de concentré. C'est ainsi que le traitement à l'ammoniac a permis de réduire la quantité de pulpe à ajouter aux pailles de 1,46 kg/j entre les lots 1 et 5 de l'essai II, dont les croîts journaliers étaient de 314 et $325 \mathrm{~g} / \mathrm{j}$, et de $1,58 \mathrm{~kg}$ (valeur comparable) entre les lots 1 et 3 de l'essai III, dont les croîts journaliers étaient de 479 et $546 \mathrm{~g} / \mathrm{j}$ (certes différents, mais non significativement).

L'adjonction de tourteau tanné, qui apportait une quantité de PDI excédentaire par rapport aux besoins théoriques nécessaires pour les croîts effectivement réalisés, n'a pas modifié significativement les croissances journalières moyennes (Tableau III) :

- augmentation non significative de $116 \mathrm{~g} / \mathrm{j}$ dans l'essai $1 /$ et de $51 \mathrm{~g} / \mathrm{j}$ dans l'essai III avec les pailles non traitées,

— augmentation de $77 \mathrm{~g} / \mathrm{j}$ et de $65 \mathrm{~g} / \mathrm{j}$ respectivement avec les niveaux élevés et faibles de pulpe dans l'essai II avec les pailles traitées,

- diminution non significative de $32 \mathrm{~g} / \mathrm{j}$ avec la paille traitée dans l'essai III.

\section{DISCUSSION}

\section{Aspects méthodologiques}

Ces trois essais visaient à préciser :

- le niveau minimum de la complémentation énergétique à apporter en plus de pailles traitées pour assurer à des génisses de 2 ans des croissances hivernales de l'ordre de $350 \mathrm{~g} / \mathrm{j}$. Ceci revient en fait à exprimer l'augmentation de la valeur alimentaire de pailles, correctement traitées à l'ammoniac, en termes d'économie de complément par rapport aux mêmes pailles non traitées; 
- l'intérêt de protéines peu dégradables dans la complémentation des pailles, traitées ou non;

- l'intérêt respectif d'une complémentation appropriée et du traitement chimique des pailles.

Certains problèmes d'ordre méthodologique doivent être examinés avant toute discussion. On sait en effet que l'appréciation des croissances journalières est d'autant plus entachée d'erreurs que ces croissances sont faibles. Malgré les précautions que nous avons prises comme les doubles pesées, ou encore les périodes pré- et post-expérimentales sur des régimes communs permettant d'uniformiser entre les lots les "poids" de contenus digestifs, les erreurs absolues commises sur la mesure du poids vif des animaux restent élevées. Les erreurs relatives qui en résultent sur l'appréciation des croissances journalières le sont encore plus. C'est ainsi que leurs coefficients de variations, très élevés dans l'essai I, sont encore de 30 à $40 \%$ dans l'essai II et ne diminuent à un niveau raisonnable (de 16 à $28 \%$ ) que dans l'essai III.

La prise en compte systématique des périodes strictement expérimentales risque parfois (ce fut le cas dans nos essais II et III) de conduire à des erreurs si on ne considère pas les circonstances dans lesquelles les essais se sont déroulés pratiquement : dans notre cas, les périodes très froides intervenues en janvier dans les essais II et III. En outre, le fait que l'œstrus et l'insémination tombaient pendant les phases expérimentales a modifié le comportement alimentaire (ou l'appétit) des animaux, comme le montrent les Figures 1a, 2a et 3a.

En outre, le fait que les génisses aient été maintenues sur aire paillée constitue une source d'erreur (estimée à 10\%) dans la mesure des consommations réelles de paille qui, dans nos résultats, sont donc légèrement sous-estimées par les lots P.NT et légèrement biaisées pour les lots pailles traitées.

Enfin un autre point compliquant l'interprétation des résultats est le fait d'avoir distribué systématiquement $1,5 \mathrm{~kg}$ de $M S$ d'ensilage (de bonne qualité) en plus de la pulpe. Cet aliment complémentaire, bien que favorisant les conditions de la cellulolyse dans le rumen (Dulphy \& Bony, 1983) a certainement réduit les quantités de pailles ingérées et, par là, l'effet propre de la pulpe déshydratée (également favorable à cette cellulolyse) dans la valorisation de rations à base de pailles. II convient donc d'interpréter les résultats de consommation et de croissance avec prudence.

Par ailleurs, la présence d'ensilage a constitué une source d'erreur dans la précision de l'évaluation de la valeur d'encombrement et de la valeur alimentaire des diverses pailles. Pour cette évaluation nous avons attribué à l'ensilage une valeur de 1,35 UEB et, aux pulpes, une valeur de 1,05 UEB.

\section{Mode de traitement}

Le traitement au four s'est essentiellement traduit par une économie d'ammoniac. Malgré une augmentation de $1 \mathrm{~kg} / \mathrm{j}$ des consommations de pailles par les génisses par rapport au traitement en tas la croissance n'a pas été modifiée (elle a même diminué mais de façon non significative). A valeur énergétique égale (mesurée sur mouton) il est alors possible que cette diminution soit due à une moins bonne utilisation de l'azote fixé. L'élévation rapide de température lors du traitement pourrait entraîner la formation de 
composés azotés moins bien utilisables par les micro-organismes du rumen.

\section{Complémentation énergétique mini- mum}

On a étudié, dans l'essai I, s'il était possible de supprimer l'apport de pulpe. La paille traitée représentait alors de l'ordre de $75 \%$ de la matière sèche totale ingérée. On sait (Dulphy et al., 1984a) que le bénéfice du traitement d'une paille sur sa valeur alimentaire est d'autant plus net que sa proportion dans la ration est élevée. Malgré cette proportion élevée, l'augmentation marquée de la digestibilité de la paille mesurée sur moutons et des quantités de paille ingérées par les génisses après traitement et, malgré la présence de $1,5 \mathrm{~kg}$ de MS d'ensilage, les croissances des génisses ont été pratiquement nulles (Tableau III). Il est donc nécessaire, pour obtenir sur des génisses de 2 ans des croissances de $350 \mathrm{~g} / \mathrm{j}$, de complémenter les P.NH$H_{3}$ avec un minimum d'énergie supplémentaire. Cette dernière étant vraisemblablement nécessaire pour permettre l'utilisation optimum par les micro-organismes du rumen et par l'animal hôte de l'azote apporté par le traitement de l'ammoniac.

L'adjonction de 2,6 kg de MS de pulpe a entraîné des croissances supérieures à celles prévues au départ. On observe cependant bien, dans ce cas, que l'augmentation de la valeur énergétique nette des pailles traitées, calculée à partir des besoins théoriques des génisses pour les performances réalisées, est plus faible (- 0,01 et $+0,17 \mathrm{UFL} / \mathrm{kg}$ MS) qu'avec P. $\mathrm{NH}_{3}$ sans pulpe $(+0,33$ et $+0,30$ UFL $/ \mathrm{kg}$ MS). Elle est également plus faible que l'augmentation de la valeur énergétique calculée à partir de la mesure de la digestibilité sur mouton $(+0,17$ UL/kg MS). Ceci confirme bien la diminution du bénéfice du traitement des pailles lorsque la part de ces dernières dans la ration diminue, même lorsque la complémentation est favorable à la cellulolyse.

Ainsi, la moins bonne valorisation de la paille traitée en présence de $2,6 \mathrm{~kg}$ de MS de pulpes par rapport à celle des pailles traitées distribuées seules doit vraisemblablement refléter, soit une mauvaise utilisation digestive liée à l'associativité négative des compléments sur la digestibilité de la paille (la $\mathrm{P} . \mathrm{NH}_{3}$ ne représentait que 30 à $35 \%$ de la ration totale et n'apportait que 38 et $33 \%$ des besoins d'entretien), soit une mauvaise utilisation de l'azote apporté par l'ammoniac du traitement; hypothèse formulée plus haut, que nous avons voulu vérifier dans les essais II et III, soit les deux phénomènes à la fois.

Dans les essais II et III nous n'avons fixé qu'à $1,7 \mathrm{~kg}$ de MS de pulpe la diminution maximale de complémentation entre les P.NT et les $\mathrm{P.NH}_{3}$ et nous n'avons pas observé de diminution de croissance. L'économie de la complémentation énergétique rendue possible par le traitement confirme celle observée par Dulphy et al. (1984b) et par pluieurs auteurs étrangers (cf. la revue de Kristensen, 1984). Elle est de l'ordre de 1,5 UFLj et permet en outre de mieux tirer parti du bénéfice du traitement. Ainsi, comme le montrent les résultats des lots 3 et 4 de l'essai II, où la réduction de la complémentation énergétique n'était qu'«intermédiaire" $(-0,5 \mathrm{~kg} / \mathrm{j}$ de pulpes de betteraves déshydratées), la croissance journalière n'a été augmentée que de $114 \mathrm{~g} / \mathrm{j}$, la paille traitée ne représentant alors (Tableau III) qu'environ $55 \%$ de la ration totale au lieu de 67 et $66 \%$ et sa valeur énergétique n'étant que de 0,46 UFL/kg de MS au lieu de 0,56 et de 0,62 
(Tableau III) avec les niveaux de complémentation plus faibles (lots 5 de l'essai II et lot 3 de l'essai III).

\section{Influence d'un apport de tourteau tanné, source de PDIA}

L'effet du tourteau tanné sur les quantités de paille ingérées, pratiquement nul ou négatif dans l'essai II, n'a été positif que dans l'essai III et surtout dans le cas des

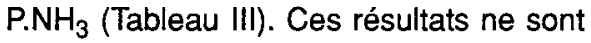
donc que partiellement en accord avec ceux de Egan (1965) et de Preston \& Leng (1984) qui avaient montré que la fourniture supplémentaire d'acides aminés au niveau de l'intestin grêle permettait d'augmenter la capacité d'ingestion des animaux grâce à leur meilleur état nutritionnel azoté. Ces derniers auteurs avaient travaillé en zone tropicale avec des animaux en croissance modérée (inférieure à $500 \mathrm{~g} / \mathrm{j}$ ) consommant des pailles traitées ou non qui représentaient au moins $75 \%$ de la ration. Les compléments facilement fermentescibles étaient constitués d'aliments énergétiques et azotés peu dégradables dans le rumen.

Dans notre cas, les protéines peu fermentescibles apportées, à même niveau énergétique total entre lots, sous forme de tourteau tanné en plus des besoins azotés théoriquement nécessaires ont entraîné, sauf dans l'essai III, une augmentation supplémentaire des croîts, certes non significative, mais intéressante.

L'intérêt du tourteau tanné a peut-être été en partie masqué par l'apport de 1,5 $\mathrm{kg}$ de MS d'ensilage de bonne qualité et par la pulpe qui apporte aussi de l'azote peu fermentescible.

Toutefois la réponse positive d'une source de PDIA sur les croissances observées dans l'essai II peut laisser penser que :

- ou bien, si les tables des besoins en PDI (INRA, 1988) sont exactes, le calcul des apports est surestimé puisqu'un apport (sous forme de PDIA) supplémentaire par rapport à ces besoins supposés couverts augmente encore les croîts. II conviendrait alors de revoir le mode de calcul des PDIE et PDIN des pailles (surtout à l'ammoniac). Cette remarque est d'ailleurs conforme à celles de Demarquilly et al. (1987) qui évoquent les problèmes liés à la mauvaise utilisation de l'azote apporté par l'ammoniac du traitement;

- ou bien, si les calculs sont corrects, les besoins azotés des animaux en croissance sont sous-estimés et les apports insuffisants par rapport aux besoins réels.

Indépendamment des hypothèses formulées ci-dessus, ces résultats méritent également d'être rapprochés des observations récentes de Silva \& Ørskov (1983), Preston \& Leng (1984), Gibb \& Baker (1987), Ramihone \& Chenost (1988) et de quelques autres auteurs. Ces dernières mettent en effet toutes en évidence l'intérêt : 1) d'un apport d'acides aminés, source de chaînes carbonées nécessaires à la néoglucogenèse qui est faible avec de tels régimes, et 2) d'autres nutriments, encore mal identifiés d'ailleurs (Silva \& Ørskov, 1988), sur la nutrition non seulement de l'animal hôte mais également des micro-organismes cellulolytiques du rumen. Un autre intérêt de l'apport d'azote lentement dégradable est de fournir l'azote et l'énergie aux micro-organismes du rumen de façon plus régulière et mieux synchronisée qu'avec une source azotée classique comme le laissent penser les travaux de Ramihone (1987).

Enfin, le fait que les pailles non traitées des essais II et III et complémentées correctement en pulpe et en tourteau de soja 
tanné permettent les mêmes croissances que les pailles traitées nous autorise à poser la question de savoir, du moins pour des pailles en l'état ayant une valeur alimentaire voisine de celles que nous avons utilisées, s'il convient de traiter ou de complémenter. $\mathrm{La}$ réponse finale appartient aux économistes car elle implique la prise en compte de nombreux autres paramètres (prix respectifs des compléments et du (des) traitement(s) utilisé(s), modification des systèmes d'élevage, investissements, etc.) et il nous est difficile, ici, de donner des éléments économiques qui seraient insuffisants. On peut toutefois penser, dans une hypothèse de traitement, que c'est le type d'animaux et le niveau des croissances recherchées qui vont déterminer le choix de la complémentation. Celle-ci pourra être minimum, du type lot 2 du 1 er essai pour des animaux adultes à l'entretien et du type lots 5 et 6 du $2^{\text {e }}$ essai pour des génisses en croissance modérée.

\section{CONCLUSION}

Le traitement des pailles à l'ammoniac, lorsqu'il est bien réussi, permet d'améliorer leur digestibilité et leur ingestibilité. II permet donc, soit d'améliorer les performances animales à même niveau de complémentation, soit d'obtenir les mêmes performances avec une complémentation réduite. En fait le bénéfice du traitement est surtout dû à l'augmentation des quantités de paille ingérées. En effet, comme nous l'avons vu dans nos essais (Tableau III), l'augmentation de la valeur énergétique des pailles (alors considérées "à l'intérieur» de la ration totale) est très variable. Cette valeur dépend de la proportion (qui ne devrait pas être supérieure à $30 \%$ ) et de la nature des concentrés (énergie fermentescible de type "paroi" et azote peu ou lentement dégradable) dans la ration qui ont, en effet, une importance déterminante sur la cellulolyse dans le rumen.

Cependant, comme le montrent nos résultats ef ceux de très nombreux auteurs (Cee - Cost 84 bis, sous presse), la valeur alimentaire d'une paille traitée, et en particulier l'augmentation des quantités qui en seront ingérées sont difficiles à prédire. Aussi, sur un plan pratique on admettra qu'un traitement correctement effectué permet à des génisses laitières de 2 ans de réaliser les mêmes croissances qu'avec des pailles non traitées tout en réduisant l'apport journalier de concentré de $1,5 \mathrm{~kg}$ à $2 \mathrm{~kg}$ (économie de 1,5 à 2 UFL).

Pour qu'un traitement se traduise par un bénéfice de cet ordre, il convient cependant d'être vigilant en ce qui concerne la nature des aliments de complément. Nous avons utilisé de l'ensilage d'herbe et des pulpes de betteraves déshydratées (aliments riches en parois rapidement digestibles) pour favoriser la cellulolyse. Toutefois des observations en exploitation de Grenet et al. (1985) montrent que des céréales classiques (orge, maïs) conduisent également aux mêmes économies. Nos essais confirment, en revanche, l'importance d'une complémentation azotée appropriée, mise en évidence jusqu'ici seulement à l'échelle du laboratoire et par un très petit nombre d'auteurs. Les observations de Silva \& Ørskov (1988) et de Ramihone \& Chenost (1988), montrent en effet l'intérêt d'une complémentation des pailles par du tourteau tanné ou, mieux, par la farine de poisson sur la nutrition (et l'activité) des micro-organismes du rumen.

II serait intéressant de compléter ces essais par d'autres faisant intervenir d'autres aliments protéiques non seule- 
ment lentement dégradables, mais apportant, comme la farine de poisson, d'autres nutriments (encore mal identifiés) indispensables aux micro-organismes du rumen. On pourrait ainsi en vérifier l'intérêt pour la complémentation, soit des pailles non traitées, soit des pailles traitées à l'ammoniac (l'effet du traitement pourrait être certainement mieux exprimé). II conviendrait bien sûr, dans ces essais, de ne complémenter les pailles qu'avec la quantité d'énergie minimum de type parois (ensilage ou pulpe seulement) de façon à obtenir des résultats mieux tranchés et plus facilement interprétables.

\section{REMERCIEMENTS}

Nous tenons à remercier Monsieur C. Demarquilly, directeur de l'unité valeur alimentaire, pour les conseils judicieux qu'il nous a apportés dans la mise en place des essais et l'exploitation des résultats.

\section{RÉFÉRENCES}

Andrieu J. \& Demarquilly C. (1987) Composition alimentaire des foins et des pailles. In : Les Fourrages Secs : Récolte, Traitement et Utilisation. (C. Demarquilly ed.) INRA, Paris, 163-182

Cee-Cost 84 bis. (1989) Proceedings of a Workshop on Evaluation of Straws in Ruminant Feeding (M. Chenost \& P. Reiniger eds), Elsevier, Amsterdam

Chenost M. (1987) Influence de la complémentation sur la valeur alimentaire et l'utilisation des mauvais foins et des pailles par le ruminant. In : Les fourrages secs : récolte, traitement et utilisation. (C. Demarquilly, ed.), INRA, Paris, 199-230

Cilenost M. \& Demarquilly C. (1985) Utilisation par les ruminants de fourrages et sous-produits à très faible valeur nutritive. In : Colloque : Optimisation de la production animale à partir de fourrages très grossiers. FAO/CEE, Genève, 21-25 janvier 1985

Chenost M., Grenet N. \& Hoden A. (1987) Utilisation des pailles par les bovins en croissance. In : Les fourrages secs : récolte, traitement et utilisation. (C. Demarquilly, ed.) INRA, Paris, pp. 319-334

Chesson A. \& Ørskov E.R. (1984) Microbial degradation in the digestive tract. In : Straws and other fibrous by-products as feed. ( $F$. Sundstøl \& E. Owen, eds.), Elsevier, Amsterdam, pp. 305-339

Cordesse R. (1987) Technologie du traitement des pailles à l'ammoniac. In : Les fourrages secs : récolte, traitement et utilisation. (C. Demarquilly, ed.), INRA, Paris, pp. 231-242

Demarquilly $C$., Chenost M. \& Ramihone B. Intérêt zootechnique du traitement des pailles à l'ammoniac. Symposium International sur l'Alimentation des Ruminants en milieu tropical humide. Guadeloupe, 2-6 juin 1987, (sous presse)

Dulphy J.P. \& Bony J. (1983) Influence de la nature de l'aliment complémentaire sur les performances de génisses recevant de la paille. Bull. Techn. CRZV Theix, INRA 52, 37-41

Dulphy J.P., Boissau J.M. \& Leblanc F. (1984a) Influence du traitement à l'ammoniac sur la valeur alimentaire de pailles distribuées à des génisses. I - Résultats d'essais. Bull. Tech. CRZV Theix, INRA, 25-32

Dulphy J.P., Komar A. \& Zwaenepoel P. (1984b) Effets comparés des traitements à l'ammoniac et à la soude sur la valeur alimentaire des fourrages pauvres. Ann. Zootech. 33, $321-342$

Duncan D.B. (1955) Multiple range and multiple $F$ test. Biometrics 11, 1-42

Egan A.R. (1965) Nutritional status and intake regulation in sheep. IV. The influence of protein supplements upon acetate and propionate tolerance of sheep fed on low quality chaffed oaten hay. Austr. J. Agric. Res. 16, 473-48

Gibb M.J. \& Baker R.D. (1987) Performances of young steers offered silage or thermo-ammoniated hay with or without a fish-meal supplement. Anim. Prod. 45, 371-381

Grenet N., Chevalier C., Roudier J., Langlois J.P., Paye M., Binet J.P., Gauthier M., Pierrard M. \& Latron J.P. (1985) Pailles traitées à l'am- 
moniac. Valorisation par des génisses et des vaches allaitantes. Miméo. N 85031

INRA (1988) Alimentation des Bovins, Ovins et Caprins (INRA ed.) 147, rue de l'Université, 75007 Paris

Kristensen V.F. (1984) Straw and other fibrous by products. In pratical rations for cattle with special reference to developped countries. In : Straws and other fibrous by-products as feed. Chap. 14-2 (F. Sunstøl \& E. Owen, eds.) Elsevier, Amsterdam, pp. 431-453

Preston T.R \& Leng R.A. (1984) Supplementation of diets based on fibrous residues and byproducts. In : Straws and other fibrous by-products as feed. (F. Sunsdtøl \& E. Owen, eds.) Elsevier, pp. 373-413

Ramihone B.R. (1987) Facteurs limitant la fermentation dans le rumen des pailles de céréales (à petits grains) traitées à l'ammoniac anhydre. Thèse, Université des Services et Techniques du Languedoc, Montpellier (France)

Ramihone B. \& Chenost M. (1988) Effet de la nature du complément protéique sur la diges- tion dans le rumen d'une paille de blé traitée ou non à l'ammoniac. Repr. Nutr. Develop. 28, 91-92

Silva S.G. \& Ørskov E.R. (1983) cités par Chesson A. \& Ørskov E.R. (1984) Microbial degradation in the digestive tract. In : Straws and other fibrous by-products as feed. ( $\mathrm{F}$. Sundstel, E. Owen eds.), Elsevier, Amsterdam, pp. 305-339

Silva A.T. \& Ørskov E.R. (1988) The effect of five different supplements on the degradation of straw in sheep given untreated barley straw. Anim. Feed Sci. Technol. 19, 289-298

Sundstøl F., Coxworth E. \& Mowat D.N. (1978) Improving the nutritive value of straws or other low quality roughages by treatment with ammonia. World Anim. Rev. FAO, 26, 13-21

Tuah A.K., Lefadeju E. \& Ørskov E.R. (1986) Rumen degradation of straw. 1 untreated and ammonia treated barley, oat and wheat straw varieties and triticale straws. Anim. Prod. 43, 261-269 